\title{
Neonatal neutrophils stimulated by group B Streptococcus induce a proinflammatory T-helper cell bias
}

\author{
Jianguo Lin ${ }^{1}$, Seema Haridas ${ }^{2}$, Stephen J Barenkamp ${ }^{2}$, Larissa Chioquetta Lorenset ${ }^{3}$, Ashley Sang Eun Lee ${ }^{2}$, \\ Benjamin T Schroeder ${ }^{2}$, Guangyong Peng ${ }^{2}$ and Joyce M Koenig ${ }^{2}$
}

BACKGROUND: Group B Streptococcus (GBS) infection causes inflammatory comorbidities in newborns. While the mechanisms remain unclear, evidence suggests that impaired innateadaptive immune interactions may be contributory. We hypothesized that GBS-stimulated neonatal neutrophils provide a milieu that may drive proinflammatory T-helper (Th) cell programming.

METHODS: Neutrophils were stimulated with Type III GBS $(\mathrm{COH} 1)$; supernatants or intact neutrophils were cocultured with $\mathrm{CD}^{+} \mathrm{T}$ cells or regulatory $\mathrm{T}$ cells (Tregs). Resulting intracellular cytokines and nuclear transcription factors were determined by multicolor flow cytometry.

RESULTS: GBS-stimulated neutrophils released soluble mediators that induced greater interleukin-17 (IL-17) responses in neonatal vs. adult $\mathrm{CD}^{+} \mathrm{T}$ cells in the absence of added polarizing cytokines. GBS-stimulated neonatal neutrophils also induced robust expression of the canonical nuclear transcription factors for Th1 (Tbet) and Th17 (IL-17) cells in CD4 ${ }^{+}$T cells. Following GBS stimulation, both intact neutrophils and neutrophil-derived mediators promoted the generation of Tregs with Th1 and Th17 characteristics.

CONCLUSION: GBS-stimulated neonatal neutrophils bias the in vitro Th differentiation program of neonatal $\mathrm{CD}^{+} \mathrm{T}$ cells and promote proinflammatory Th1 and Th17 phenotypes in Tregs. Our data suggest that developmental modifications of innate-adaptive immune cross-talk mechanisms may contribute to the inflammatory complications associated with neonatal GBS infection.

G roup B Streptococcus (Streptococcus agalactiae, GBS) is a Gram-positive bacterium that frequently colonizes the intestines and lower genital tract. GBS is particularly pathogenic in immunocompromised individuals $(1,2)$, and GBS infections are a leading cause of fetal and infant death in the United States and around the world (3). Imbalanced fetal and neonatal immune responses facilitate the inflammatory morbid complications and mortality associated with GBS infection (4). The exact mechanism(s) by which GBS induces neonatal inflammatory responses remain(s) enigmatic; however, neutrophils appear to be a key cellular component in this process (5). The findings of neutrophil infiltration in the fetal placenta and in preterm neonatal lungs were also particularly striking in association with fatal intrauterine GBS infection (6).

Neutrophils play a sentinel role in modulating immune responses to invading pathogens (reviewed in ref. (7)). Although neonatal neutrophils exhibit intrinsic functional impairments that increase host susceptibility to infections (reviewed in ref (8)), paradoxically they can also exhibit exaggerated inflammatory responses to microbial stimulation $(9,10)$. Neutrophils collaborate with the adaptive immune T-helper type 17 (Th17) cells to amplify inflammatory processes that may be protective against infections or that can foster the pathogenesis of inflammatory disorders (11). Although the contributions of neutrophil-Th17 cell interactions to neonatal inflammation have not been described, evidence in humans and mice indicates a neonatal capacity for robust Th17 responses (12), particularly under conditions also associated with exacerbated neutrophil responses $(13,14)$. Thus, neutrophil-Th17 interactions likely contribute to inflammatory morbidities in young infants, including those associated with GBS infections.

Studies have shown the induction of Th1- or Th17-type immune responses during GBS infection and colonization $(15,16)$. Inflammatory monocytes can promote Th17 cell propagation by releasing the polarizing cytokines, interleukin$1 \beta$ (IL-1 $\beta$ ) and IL-6 (17), which are also produced by neutrophils (reviewed in ref. (18)). However, whether neonatal neutrophils have a direct role in the generation of Th1 and Th17 cells, particularly in the context of infection, has not been reported. The present studies were designed to test our hypothesis that GBS can stimulate neonatal neutrophils to drive inflammatory adaptive immune responses.

\section{METHODS}

\section{Ethics Statement}

This study was performed under an approved protocol and in strict accordance with the guidelines and policies of the Institutional Review Board for Human Studies of Saint Louis University.

\footnotetext{
${ }^{1}$ Department of Neurology, Guangdong No. 2 Provincial People's Hospital, Guangzhou, China; ${ }^{2}$ Saint Louis University, St Louis, Missouri; ${ }^{3}$ Federal University of Grande Dourados, Dourados Brazil. Correspondence: Joyce M. Koenig (joyce.koenig@health.slu.edu)

Received 18 March 2017; accepted 6 October 2017; advance online publication 6 December 2017. doi:10.1038/pr.2017.272
} 


\section{Blood Samples}

Human umbilical cord blood (CB) samples from healthy full term newborns were obtained with parental consent immediately after delivery for all neutrophil studies. For other studies, anonymous $\mathrm{CB}$ samples (less than $12 \mathrm{~h}$ post-delivery) were obtained from the SSM Health St. Louis Cord Blood Bank. Consented healthy adult volunteers were used as controls in some studies.

\section{Neutrophil and $\mathrm{CD}^{+}{ }^{+} \mathrm{T}$ Cell Isolation}

Mononuclear cells and neutrophils were isolated by density centrifugation from $\mathrm{CB}$ or adult peripheral blood using FicollPaque PLUS gradient (GE Healthcare). $\mathrm{CD} 4^{+} \mathrm{T}$ cells or naïve $\mathrm{CD} 4^{+}$ $\mathrm{T}$ cells were isolated by negative selection (EasySep ${ }^{\mathrm{TM}}$ Human $\mathrm{CD} 4^{+}$ $\mathrm{T}$ cell enrichment kit or EasySep ${ }^{\mathrm{TM}}$ Human Nä̈ve $\mathrm{CD}^{+}{ }^{+} \mathrm{T}$ Cell Isolation Kit, StemCell Technologies, Vancouver, Canada) according to the manufacturer's instructions. Tregs and T effector cells (Teff) were isolated from purified $\mathrm{CD}^{+}$cells by positive selection (EasySep $^{\text {TM }}$ Human CD25 Positive Selection Kit, StemCell Technologies). Neutrophil purity, determined by H\&E stain (Dif-Quik, Sigma, St. Louis), was > 95\%. Neutrophil viability, determined by trypan blue staining, was $>94 \%$ following isolation procedures, while $60-70 \%$ viability was observed following a $1 \mathrm{~h}$ incubation with heat-killed (HK) GBS.

\section{Group B Streptococcus}

$\mathrm{COH} 1$, a virulent serotype III GBS strain from a neonate with sepsis (a generous gift of Dr Morven Edwards), was grown to log phase in Todd-Hewitt broth (Difco, Detroit, MI), washed, and re-suspended in phosphate-buffered saline (PBS) (19). To obtain HK-GBS for studies, bacteria diluted in PBS were incubated at $56^{\circ} \mathrm{C}$ for $30 \mathrm{~min}$; bacterial death was confirmed by re-plating HK-GBS samples. Aliquots of the HK-GBS stock solution of these GBS isolates were stored at $-70^{\circ} \mathrm{C}$.

\section{GBS Stimulation Studies}

Isolated neutrophils $\left(2.5 \times 10^{6}\right.$ cells $\left./ \mathrm{mL}\right)$ suspended in RPMI 1640, $10 \%$ FBS were co-cultured at $37^{\circ} \mathrm{C}, 5 \% \mathrm{CO}_{2}$ for $1 \mathrm{~h}$ in media alone or in the presence of heat-killed GBS (hereafter referred to as "GBS") at a ratio of 10 bacteria per neutrophil (20). Harvested cell-free culture supernatants (unstimulated neutrophil-conditioned medium, NCM; or GBS-stimulated neutrophil-conditioned medium, NCM-GBS) were stored at $-80^{\circ} \mathrm{C}$ until bioassay. For cell-cell (neutrophil-CD4 ${ }^{+}$ $\mathrm{T}$ cell) contact experiments, neutrophils cultured in media alone (PMN) or in the presence of GBS (PMN-GBS) were pelleted then resuspended in complete $\mathrm{T}$ cell medium $(2 \mathrm{mM}$ glutamine, $50 \mu \mathrm{M}$ $\beta$-mercaptoethanol, $10 \%$ heat-inactivated human $\mathrm{AB}$ type serum, $100 \mathrm{U}$ penicillin $/ 100 \mu \mathrm{g}$ streptomycin/mL) before addition to $\mathrm{CD} 4^{+} \mathrm{T}$ cell cultures. For studies to compare the effects of neutrophil supernatants and intact neutrophils on $\mathrm{CD}^{+}$target cells, GBSstimulated or control CB neutrophils, or the resulting $\mathrm{CB}$ neutrophil supernatants, were added to autologous $\mathrm{CB} \mathrm{CD} 4^{+} \mathrm{T}$ cell cultures in same-day experiments, as described below. To assess a direct effect of GBS, bacteria $\left(2.5 \times 10^{7} / \mathrm{mL}\right)$ suspended in media were cultured for $1 \mathrm{~h}$; cell-free bacterial supernatants were stored at $-80^{\circ} \mathrm{C}$ until bioassay.

\section{$\mathrm{CD}^{+}{ }^{\mathrm{T}}$ Cell Co-Cultures}

Purified $\mathrm{CD}^{+} \mathrm{T}$ cells $\left(2 \times 10^{6}\right.$ cells $)$ were suspended in media and cultured either in media alone, or in media containing $50 \%$ (vol $/ \mathrm{vol}$ $\%)$ of NCM or 50\% NCM-GBS. For cell-cell contact studies, neutrophils suspended in media were co-cultured with $\mathrm{CD} 4^{+} \mathrm{T}$ cells at a 2:1 ratio. All $\mathrm{CD} 4^{+} \mathrm{T}$ cells were cultured on 24 well plates coated with anti-CD3 $\mathrm{Ab}(2 \mu \mathrm{g} / \mathrm{mL})$ and in the presence of IL-2 $(50 \mathrm{U} / \mathrm{mL})$ at $37^{\circ} \mathrm{C}, 5 \% \mathrm{CO}_{2}$ for 3-6 d (21). For $6 \mathrm{~d}$ cultures, culture media was refreshed on d 3 with starting media containing IL-2.

\section{Antibodies}

The following fluorochrome-labeled $\mathrm{Ab}$ and their respective isotype IgG controls (purchased from Becton-Dickinson, NJ, unless otherwise indicated) were used for surface or intracellular staining of cells: CD4-V500 (clone RPA-T4), CD25-BV605 (2A3), IFN $\gamma$-FITC (B27), IL-17A-AF700 (N49-653), FoxP3-APC (eBiosciences, clone PCH101), Tbet-V450 (O4-46), GATA-3-PECy7 (L50-823), and ROR $\gamma t-P E(Q 21-559)$.

\section{Flow Cytometry and T helper (Th) Cell Subset Analyses}

To identify Th-specific intracellular cytokines and nuclear transcription factors, at the end of culture $\mathrm{CD}^{+} \mathrm{T}$ cells were stimulated with $50 \mathrm{ng} / \mathrm{mL}$ PMA and $1 \mu \mathrm{g} / \mathrm{mL}$ ionomycin for $1 \mathrm{~h}$ prior to the addition of $5 \mu \mathrm{g} / \mathrm{ml}$ of Brefeldin A (Golgi-Stop ${ }^{\circledR}, \mathrm{BD}$ ), then incubated for an additional $4 \mathrm{~h}$ at $37^{\circ} \mathrm{C}$. Stimulated cells stained for surface antigens (CD4, CD25) were then fixed and permeabilized (anti-human FoxP3 staining set, eBioscience) as per the manufacturer's instructions. Permeabilized cells were stained for intracellular antigens using fluorochrome-labeled $\mathrm{mAbs}$ or $\mathrm{mAb}$ type-specific, fluorochromelabeled IgG controls. Samples were acquired within $24 \mathrm{~h}$ of staining using a 16-color BD LSRII Flow Cytometer. Acquired samples were analyzed using the FlowJo 7.2.2 software (Tree Star, Ashland, OR). Within the gated CD4 ${ }^{+} \mathrm{T}$ cell population, Th1 cells were identified by their expression of Tbet and/or IFN $\gamma$, and Th17 cells were identified by their expression of ROR $\gamma \mathrm{t}$ and/or IL-17A. Tregs were identified as $\mathrm{CD} 4^{+} \mathrm{CD} 25^{+} \mathrm{Foxp}^{+}$or $\mathrm{CD} 4^{+} \mathrm{CD} 25^{+}$populations; the $\mathrm{CD} 4^{+} \mathrm{CD} 25^{-}$populations represented $\mathrm{T}$ effector cells (Teff).

\section{Statistical Analysis}

Experimental data were compared using one-way ANOVA (multiple comparisons) or the paired Student $t$ - test as appropriate using Prism v6.03 (GraphPad Software, Inc., La Jolla, CA). A $P$ value $<0.05$ was considered to be significant.

\section{RESULTS}

\section{Soluble Mediators Released by GBS-Stimulated Neutrophils Induce higher Frequencies of IL-17 $7^{+}$CD4 Cells in Neonatal vs. Adult Cultures}

To determine if GBS stimulation of neonatal and adult neutrophils might induce differential responses in target $\mathrm{CD} 4^{+} \mathrm{T}$ cells, neonatal or adult naïve $\mathrm{CD} 4^{+} \mathrm{T}$ cells (Figure 1a, b) were incubated with supernatants of autologous GBSstimulated neutrophils, and the resulting Th phenotypes were identified by intracellular staining and flow cytometric analysis. For these and all subsequent studies, a culture period of 6 days was chosen to maximize Th17 responses in cultured $\mathrm{CD}^{+} \mathrm{T}$ cells, based on preliminary data and our previous work (21). As shown (Figure 1a), supernatants of GBS-stimulated neutrophils induced the expression of the Th1 cytokine, interferon- $\gamma$ (IFN $\gamma$ ), in both neonatal and adult cocultures. Although there was a trend toward higher IFN $\gamma^{+}$ $\mathrm{CD}^{+}{ }^{+} \mathrm{T}$-cell frequencies in neonatal cultures, this difference did not reach significance $(P=0.08)$. In contrast, supernatants of GBS-stimulated neutrophils enhanced the frequencies of $\mathrm{CD}^{+} \mathrm{T}$ cells that expressed the Th17 cytokine, IL-17A (hereafter referred to as IL-17), by nearly fourfold in neonatal vs. adult cultures (Figure 1b). In subsequent studies (Figure 1c,d), the effects of coculturing supernatants of unstimulated or GBS-stimulated neonatal neutrophils with $\mathrm{CD} 4^{+} \mathrm{T}$ cells on intracellular cytokine expression were compared. While only GBS-stimulated neutrophils released mediators that induced the expression of IFN $\gamma$ (Figure 1c), supernatants from both unstimulated and GBS-stimulated neutrophils induced IL-17 


\section{Neonatal neutrophils and CD4 cells $\quad$ Articles}

a

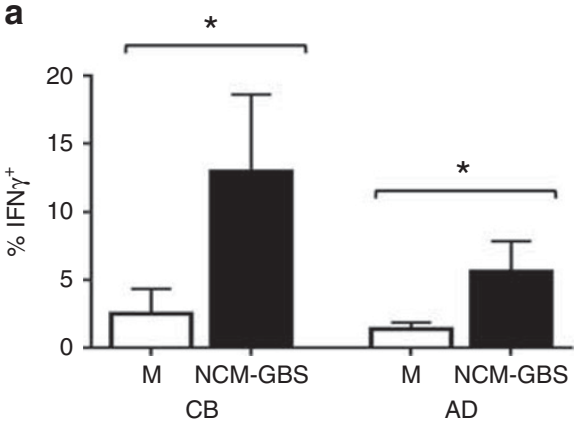

C

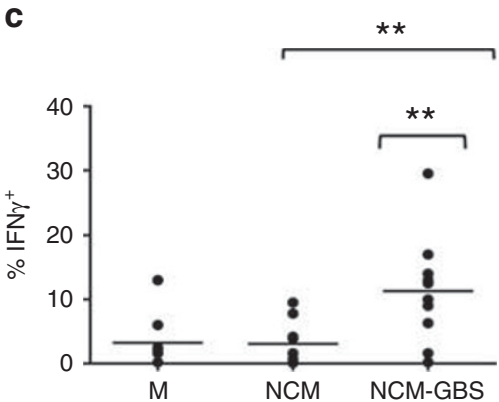

b

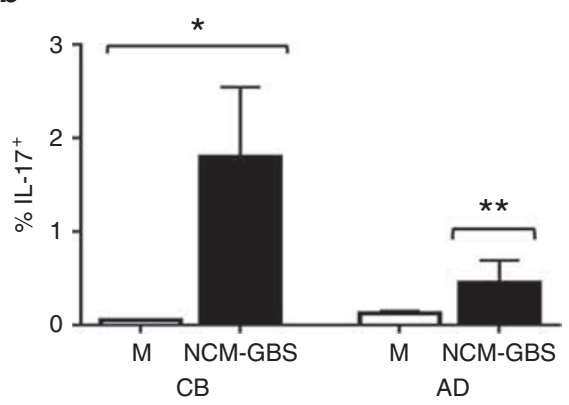

d

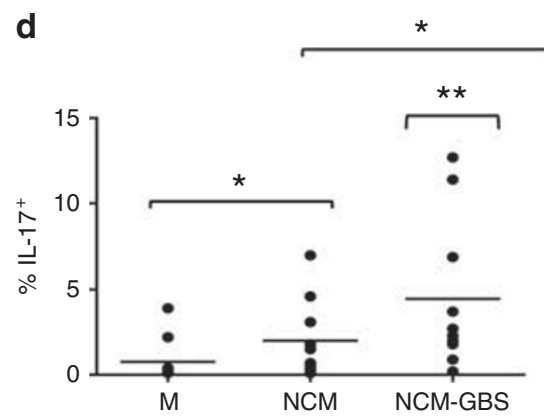

Figure 1. Group B Streptococcus (GBS)-stimulated neonatal neutrophils release soluble mediators that enhance interferon- $\gamma$ (IFN $\gamma$ ) and interleukin-17 (IL-17) expression in neonatal CD4 ${ }^{+} \mathrm{T}$ cells. (a and b) Naïve $\mathrm{CD} 4^{+} \mathrm{T}$ cells from term neonates (cord blood (CB)) or from healthy adult (AD) donors were cocultured in anti-CD3-coated plates in complete T-cell medium ( $\mathrm{M}$, white bars), or in $\mathrm{M}$ containing a $50 \%$ (vol/vol\%) concentration of supernatants of respective CB or AD neutrophils stimulated with heat-killed GBS (neutrophil-conditioned medium (NCM)-GBS, black bars). For these and all subsequent studies, IL-2 $(50 \mathrm{U} / \mathrm{ml})$ was added to cultures on day 0 and again on day 3 when media were refreshed. After a 6-day incubation, cells were stimulated before staining for intracellular cytokine analysis by flow cytometry, as described in Methods. Shown are mean frequencies of $\mathrm{CB}$ and $\mathrm{ADCD} 4^{+}$populations that coexpressed either (a) IFNy or (b) IL-17 in the presence of M or NCM-GBS. Data represent paired CB and AD studies, $n=5 ; X \pm$ SEM. ${ }^{*} P<0.05$, NCM-GBS vs. M; ${ }^{* *} P<0.05$, AD NCM-GBS vs. CB NCM-GBS. (c and d) Frequencies of CD4 ${ }^{+} \mathrm{T}-$ cell populations that expressed (c) IFNY or (d) IL-17 when cultured in the presence of M only, NCM, or NCM-GBS. Scatter-plot data represent the means of 10 individuals, replicate donor samples; $X \pm$ SEM. ${ }^{*} P<0.05$, NCM vs. M; NCM-GBS vs. NCM; ${ }^{* *} P<0.01$, NCM-GBS vs. M; NCM-GBS vs. NCM.

expression (Figure 1d), although this effect was greater for GBS-stimulated neutrophils.

\section{GBS-Stimulated Neonatal Neutrophils Release Factors that Induce Th1-, Th17-, and Treg-Specific Markers in Neonatal CD4 ${ }^{+}$ T Cells}

To assess the effects of neutrophil-derived soluble mediators on the expression of Th1 and Th17-related nuclear transcriptions factors, in the next series of studies neonatal $\mathrm{CD}^{+} \mathrm{T}$ cells were cocultured with supernatants of unstimulated or GBS-exposed neonatal neutrophils, or in media only. Coculture with supernatants of GBS-stimulated but not unstimulated neutrophils induced expression of the canonical Th1 nuclear transcription factor, Tbet (Figure 2a). Neither unstimulated nor GBS-stimulated neutrophil supernatants induced significant expression of GATA-3, the Th2 nuclear transcription factor, although the latter showed a positive trend $(P=0.07)$ (Figure 2a). In contrast, supernatants from both unstimulated and GBS-exposed neutrophils robustly induced $\mathrm{CD}^{+}$T-cell expression of the respective master nuclear transcription factors for Th17 and regulatory $\mathrm{T}$ cells (Tregs), ROR $\gamma$ t and FoxP3 (Figure 2c,d). In both cases, supernatants of GBS-stimulated neutrophils had the greatest effects relative to those of unstimulated neutrophils.
GBS-Stimulated Neonatal Neutrophils Promote the Generation of Neonatal Treg Cell Populations that Coexpress Th1- and Th17-Type Cytokines

Based on the observed induction of FoxP3 expression in $\mathrm{CD}^{+} \mathrm{T}$ cells by supernatants of neonatal GBS-stimulated neutrophils, we next sought to determine if GBS-stimulated neonatal neutrophils might also influence the generation of neonatal $\mathrm{CD}^{+} \mathrm{CD} 25^{+} \mathrm{FoxP}^{+}$Tregs. As shown (Figure 3a), neonatal $\mathrm{CD}^{+}{ }^{+} \mathrm{T}$-cell cultures containing supernatants of unstimulated or GBS-exposed neutrophils increased the mean frequencies of $\mathrm{CD} 4^{+} \mathrm{CD} 25^{+}$Foxp $3^{+}$cell populations, with the greatest effect observed for GBS-stimulated neutrophils. In subsequent studies, neonatal $\mathrm{CD}^{+} \mathrm{T}$ cells were cultured either with supernatants of GBS-stimulated neonatal neutrophils or the intact GBS-stimulated neutrophils. As shown (Figure 3b), both supernatants and intact neutrophils induced Treg populations in autologous $\mathrm{CD}^{+} \mathrm{T}$ cell populations. However, GBS-stimulated neutrophil supernatants induced a moderately but significantly greater response in $\mathrm{CD}^{+} \mathrm{T}$ cells than that observed following their contact with GBS-stimulated neutrophils.

We recently reported elevated frequencies of Tregs with Th17-type characteristics in preterm neonates exposed to 

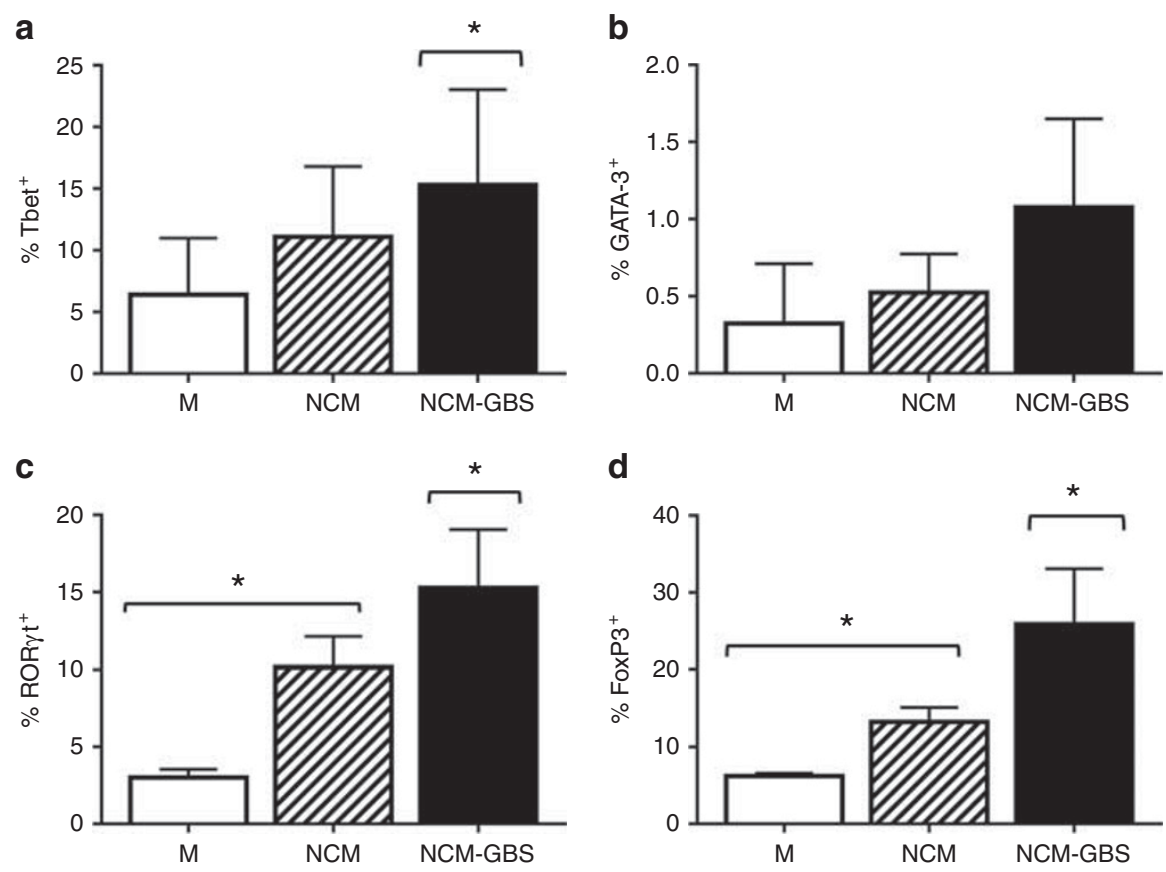

Figure 2. Soluble mediators released by neonatal neutrophils induce Tbet and RORyt expression in $\mathrm{CD} 4^{+} \mathrm{T}$ cells. Neonatal CD4 ${ }^{+} \mathrm{T}$ cells were cultured in the presence of $\mathrm{M}$ alone (white bars) or $\mathrm{M}$ containing $50 \%$ (vol/vol\%) of culture supernatants from unstimulated (neutrophil-conditioned medium (NCM), hatched bars) or GBS-stimulated neonatal neutrophils (NCM-GBS, black bars). Shown are the proportions of cultured CD4 ${ }^{+} \mathrm{T}$ cells that expressed (a) Tbet, (b) GATA-3, (c) RORyt, and (d) FoxP3. Data represent the results of replicate studies from CB donors $(n=4) ; X \pm$ SEM. ${ }^{*} P<0.05$, NCM vs. M; NCM-GBS vs. M.

a

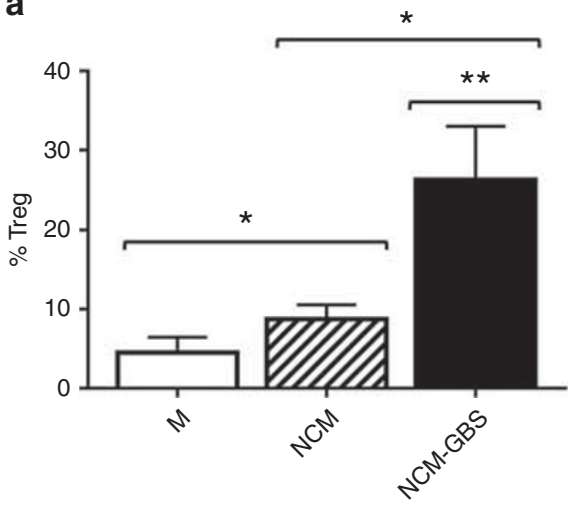

b

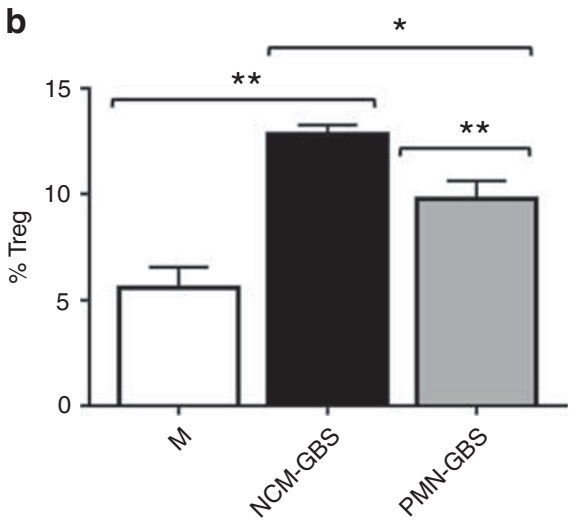

Figure 3. Group B Streptococcus (GBS)-stimulated neonatal neutrophils promote regulatory $T$ cell (Treg) generation via soluble mediators and cellcell contact. (a) Neonatal CD4 ${ }^{+} \mathrm{T}$ cells were cultured for 6 days in the presence of $\mathrm{M}$ alone (white bars) or in $\mathrm{M}$ containing (50\% vol/vol) of unstimulated (neutrophil-conditioned medium (NCM), hatched bars) or GBS-stimulated (NCM-GBS, black bars) neutrophil supernatants. (b) Neonatal $\mathrm{CD}^{+} \mathrm{T}$ cells were cultured in M (white bar), in 50\% NCM-GBS (black bar), or in the presence of intact GBS-stimulated cord blood (CB) neutrophils (polymorphonuclear leukocytes (PMN)-GBS, 2:1 PMN:CD4 ratio, gray bar). For both groups of studies, the proportions of $C D 4^{+} C D 25^{+}$Foxp3 $3^{+}$Treg cells in gated $\mathrm{CB} \mathrm{CD4}{ }^{+} \mathrm{T}$ cells were determined by flow cytometric analysis of three to five $\mathrm{CB}$ samples; $X \pm \mathrm{SEM}$. ${ }^{*} P<0.05 ;{ }^{* *} P<0.01, \mathrm{NCM}-\mathrm{GBS}$ vs. M; PMN-GBS vs. M.

histologic chorioamnionitis, a neutrophil-driven inflammation of the placenta (16). Thus, we next sought to determine if GBS-stimulated neonatal neutrophils release factors that promote the generation of Treg cells with an inflammatory phenotype in vitro. In parallel studies, culture supernatants from both unstimulated and GBS-stimulated neonatal neutrophils increased the frequencies of $\mathrm{CD} 4^{+} \mathrm{CD} 25^{+} \mathrm{FoxP} 3^{+}$ Tregs that coexpressed IFN $\gamma$ (Figure 4a,c) or IL-17 (Figure 4b,c). As was observed in related studies, soluble mediators derived from GBS-stimulated neutrophils had the more striking effect on the generation of Tregs with inflammatory cytokine expression compared with those of unstimulated neutrophils. 
a

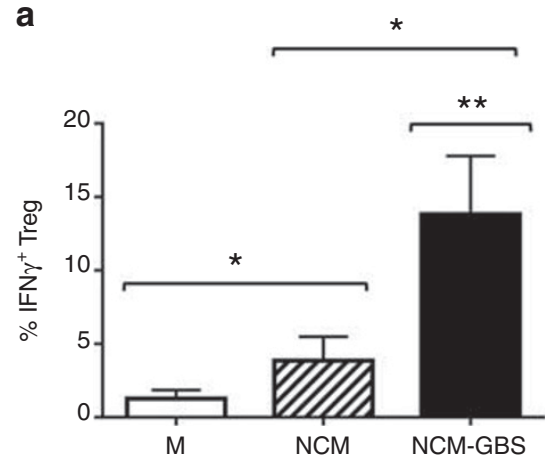

b

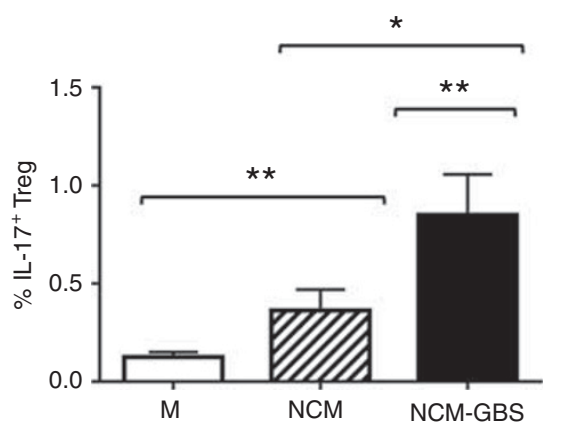

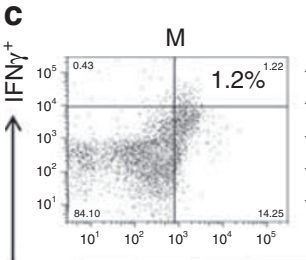
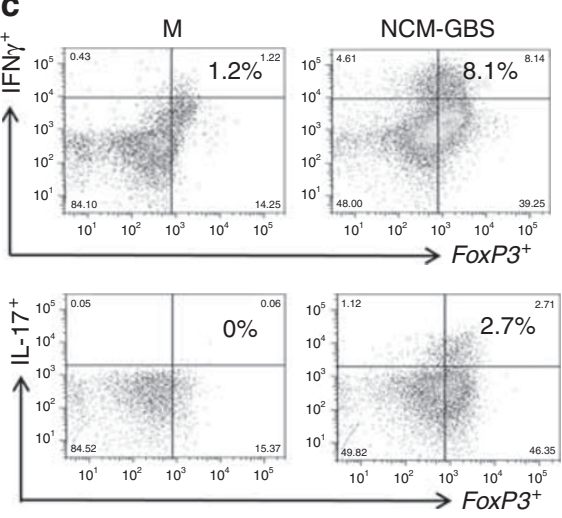

Figure 4. Group B Streptococcus (GBS)-stimulated neonatal neutrophils release soluble mediators that promote the generation of interferon- $\gamma$ -

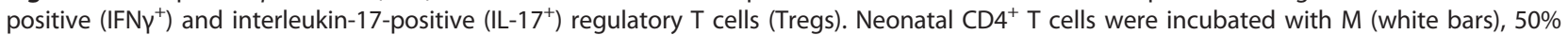
neutrophil-conditioned medium (NCM) (hatched bars), or 50\% NCM-GBS (black bars). The intracellular contents of (a and c) IFNY and (b and c) IL-17 were determined in stimulated $\mathrm{CD}^{+} \mathrm{CD} 25^{+} \mathrm{Foxp}^{+}$cell populations by flow cytometry. Data are representative of replicate studies of eight cord blood (CB) donors. $X \pm$ SEM. ${ }^{*} P<0.05$; ${ }^{* *} P<0.01, \mathrm{NCM}-\mathrm{GBS}$ vs. M; NCM vs. M. (c) Dot plots show the proportionate expression of IFN $\gamma^{+} \mathrm{FoxP3}{ }^{+}$or IL-17 ${ }^{+}$ FoxP3 $3^{+}$populations in $\mathrm{CD}^{+} \mathrm{T}$ cells following coculture with complete tissue-culture medium (M) or NCM-GBS in a representative study from one $\mathrm{CB}$ donor.
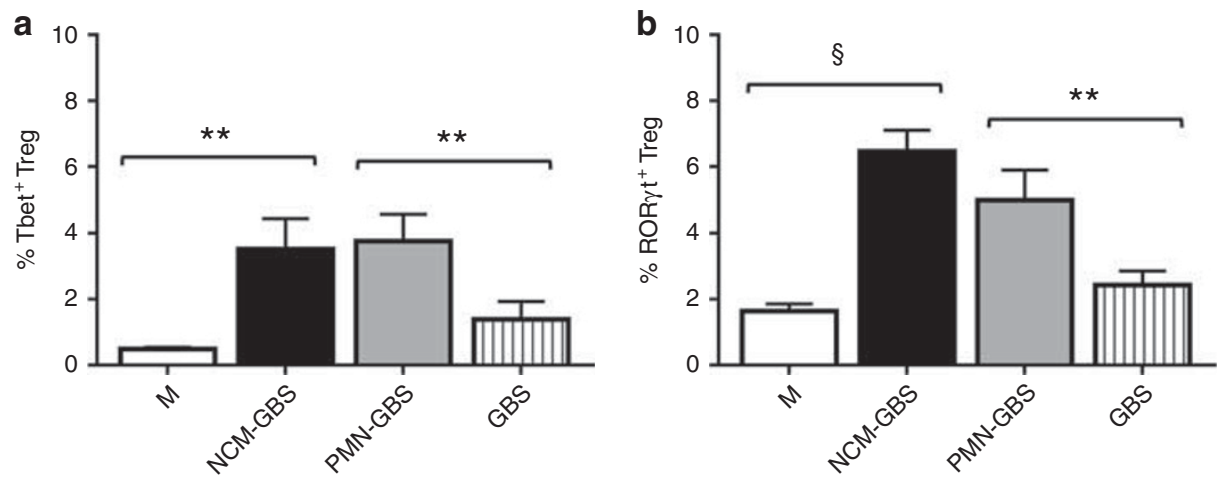

Figure 5. Group B Streptococcus (GBS)-stimulated neonatal neutrophils induce regulatory T cell (Treg) coexpression of Tbet and RORyt. Purified neonatal $\mathrm{CD} 4^{+} \mathrm{CD} 25^{+}$Tregs were cultured in the presence of $\mathrm{M}$ (white bars), 50\% neutrophil-conditioned medium (NCM)-GBS (black bars), intact GBSstimulated neutrophils (PMN-GBS, 2:1 ratio, grey bars), or cell-free supernatants of cultured GBS (GBS, striped bars). Intracellular expression levels of the nuclear transcription factors (a) Tbet and (b) RORyt were determined by flow cytometric analysis. Data represent the results of replicate studies of autologous Treg cells and neutrophils from five separate donors; $X \pm \mathrm{SEM}$. ${ }^{* *} P<0.01 ;{ }^{\S} P<0.001$.

GBS-Stimulated Neonatal Neutrophils and Derived Mediators Enhance Treg Cell Coexpression of Tbet and RORyt

We next sought to determine whether in addition to the de novo generation of Tregs with inflammatory properties, GBSstimulated neutrophils might also promote phenotypic alterations of existing Treg populations. To achieve this, we cocultured purified neonatal $\mathrm{CD} 4{ }^{+} \mathrm{CD} 25^{+}$Tregs with supernatants of autologous GBS-stimulated neutrophils or the resulting intact GBS-stimulated neutrophils, or with supernatants of GBS bacteria (Figure 5). As shown, both GBSstimulated neutrophil supernatants and intact GBSstimulated neutrophils promoted marked enhancements in Treg coexpression of Tbet (Figure 5a) and ROR $\gamma$ t (Figure 5b). In contrast, coincubation of neonatal $\mathrm{CD}^{+} \mathrm{T}$ cells with GBS supernatants alone did not significantly increase Treg coexpression of Tbet $(P=0.06)$ or ROR $\gamma \mathrm{t}(P=0.12)$ over that in media only.

\section{GBS-Stimulated Neonatal Neutrophils Release Mediators that Induce IFNy or IL-17 Expression in Neonatal Treg and Teff Populations}

To determine if neonatal Treg cells might be particularly susceptible to neutrophil-mediated induction of Th1and Th17-type responses, we compared the effects of supernatants of unstimulated or GBS-stimulated neonatal neutrophils on the intracellular expression of IFN $\gamma$ and IL-17 in neonatal naïve $\mathrm{T}$ effector cell (Teff) $\left(\mathrm{CD} 4^{+} \mathrm{CD} 25^{-}\right)$and Treg $\left(\mathrm{CD}^{+} \mathrm{CD} 25^{+}\right)$populations (Figure 6). As shown, coculture with GBS-stimulated but not unstimulated neutrophil supernatants induced coexpression of both IFN $\gamma$ 


\section{Articles | Lin et al.}

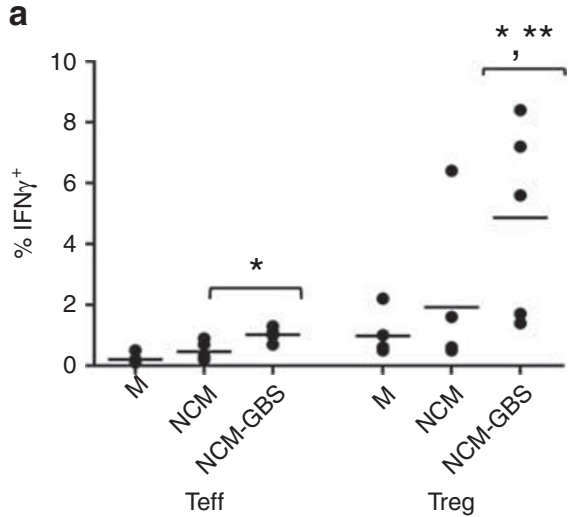

b

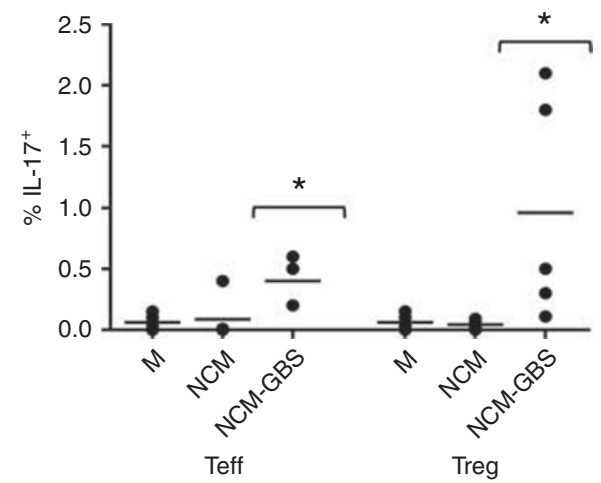

Figure 6. Group B Streptococcus (GBS)-stimulated neonatal neutrophils release mediators that induce interferon- $\gamma$ (IFN $\gamma$ ) and interleukin-17 (IL-17) expression in neonatal regulatory T cells (Tregs) and T effector cell (Teff). Neonatal CD4 $4^{+}$cells were separated into CD25 $5^{-}$(Teff) and CD25 $5^{+}$(Treg) populations by immunomagnetic selection. Cells were cultured in the presence of $\mathrm{M}$, neutrophil-conditioned medium (NCM) (50\%), or NCM-GBS $(50 \%)$, and then stimulated and stained for intracellular IFNY (a) and IL-17 (b) analysis. Data represent the results of five separate studies; $X \pm$ SEM. ${ }^{*} P<0.05, \mathrm{NCM}-\mathrm{GBS}$ vs. $\mathrm{M} ;{ }^{* *} P=0.02, \mathrm{CD} 25^{+}$vs. $\mathrm{CD} 25^{-}$cell populations.

(Figure 6a) and IL-17 (Figure 6b) in Teff and Treg cell populations. A higher mean frequency of IFN $\gamma^{+}$cells was observed in Treg vs. Teff populations (Figure 6a). In contrast, frequencies of $\mathrm{IL}-17^{+}$Treg cells were not significantly different when compared with $\mathrm{IL}-17^{+}$Teffs $(P=0.11)$ (Figure 6b).

\section{DISCUSSION}

The present studies were designed to assess the potential effects of GBS-stimulated neonatal neutrophils on the generation of inflammatory $\mathrm{CD} 4{ }^{+} \mathrm{T}$-cell populations. We now report that GBS stimulation of neonatal neutrophils induces robust Th1- and Th17-type responses in neonatal $\mathrm{CD} 4{ }^{+} \mathrm{T}$-cell and Treg populations through mechanisms that can involve cell-cell contact and soluble mediators.

We observed that GBS-stimulated neonatal neutrophils, and to a lesser degree unstimulated neutrophils, biased the CD4 differentiation program toward the generation of Th1and Th17-type cells. The inductive effects of GBS-stimulated neutrophils on expression levels of IL-17 were most striking in neonatal $\mathrm{CD}^{+}$T-cell cultures relative to those of adults. These findings are consistent with studies showing that GBS more strongly promotes inflammatory responses in neonatal monocytes relative to those of adults $(22,23)$. The higher IL-17 expression levels we observed in neonatal $\mathrm{CD} 4^{+} \mathrm{T}$ cells were not entirely surprising given the intrinsic Th17 bias previously reported in neonates (12). However, the capacity of GBS-stimulated neonatal neutrophils to promote Th17-type responses in target $\mathrm{CD} 4^{+} \mathrm{T}$ cells, without the assistance of exogenous Th17-propagating cytokines, was a novel finding. We also determined inductive effects of GBS-stimulated neonatal neutrophils on IFN $\gamma$ expression, findings supportive of potent neonatal Th1-type immune responses to microbial stimulation (24). In contrast, GBS-stimulated neonatal neutrophils induced a nominal effect on the expression of the Th2 transcription factor, GATA-3. Although neonates characteristically exhibit predominantly Th2-type immunity (reviewed in ref. (25)), Th2 responses may be suppressed under conditions that promote Th17 cells (26). We also did not find an independent effect of GBS bacteria on the induction of Th1 or Th17 responses. Our combined in vitro observations suggest that GBS promotes proinflammatory responses in neonates in part through actions mediated by targeted neutrophils.

Neonatal neutrophil-derived soluble mediators were also found to promote the propagation of Tregs in $\mathrm{CD} 4^{+} \mathrm{T}$-cell populations and to modulate the proportions of proinflammatory Tregs that coexpressed IFN $\gamma^{+}$or IL- $17^{+}$, independent of a requirement for cell contact. These observations are consistent with a recent study showing that signaling via TLR2 promotes the generation of Th17-type Tregs in the absence of antigen-presenting cells (27), which has relevance to our present findings given that GBS-mediated inflammatory signaling is driven in part by TLR2 activation (28). We also determined that GBS-stimulated neonatal neutrophils directly enhanced the in vitro generation of Treg cell populations via physical contact with $\mathrm{CD} 4^{+} \mathrm{T}$ cells, similar to findings of an inductive effect of neutrophil-CD4 cell contact on Th1 and Th17 differentiation in studies of adult mice and humans $(29,30)$.

Our present studies have identified a unique role of neonatal neutrophils in the induction of Th cell populations with protective and inflammatory potential in the context of GBS infection. While it is well established that Th1-type responses are critical in protecting the neonatal host against infections, the protective role of Th17 cells in neonates is less well defined (25). The present studies provide additional evidence of the neonatal capacity to increase potentially protective Th1 responses to infectious stimuli, particularly through TLR2 signaling mechanisms $(24,28)$. Our findings of enhanced generation of IL-17-producing immune cells in the context of a GBS stimulus also suggest their contribution to 
the neonatal host response to infection (31). However, although IL-17 is critical to protective immunity, recent studies in neonates also suggest its role in potentiating sepsisrelated inflammatory morbidity in neonates through interactions with IL-18 and in mediating the pathogenesis of necrotizing enterocolitis (32,33). Furthermore, cross-talk between neutrophils and Th17 cells can amplify injurious inflammatory responses (11), although the specific contribution of this mechanism to neonatal inflammation is unclear. In addition, our observation that GBS-stimulated neutrophils enhanced frequencies of ROR $\gamma \mathrm{t}^{+}$Tregs suggests a potentially pathogenic phenotype, as ROR $\gamma \mathrm{t}$ expression coincides with loss of Treg-suppressive function and facilitates inflammatory Treg-Th17 conversion (34). Pertinently, the TLR2 signaling associated with GBS infection (28) could potentially drive the generation of Th17-type Tregs with diminished suppressive capacity (27). Whether the Tregs induced by GBS-stimulated neutrophils exhibit primarily anti-inflammatory or pathogenic function in neonates is an important question that merits further investigation.

GBS infection and other related inflammatory disorders are associated with abundant infiltrative tissue neutrophils $(5,6)$ that could potentially colocalize with Th17 cells (11). These observations and our present studies point to neutrophils as an important environmental source of Th1- and Th17polarizing cytokines. While the specific neutrophil-derived mediators contributing to Th1 and Th17 responses were not examined in the present studies, existing evidence provides some important clues. During microbial infection, neutrophils release IL-12 and IFN $\gamma$, cytokines that promote the propagation of Th1 cells (18). In a model of GBS-induced peritonitis, neutrophils were also found to be the primary producers of IL-1 $\beta$ (5), a key Th17-propagating cytokine (35). Neonatal neutrophils robustly released IL-1 $\beta$ under inflammatory conditions (9) and following exposure to peptidoglycan, the major exoskeleton component of Gram-positive bacteria (36). Thus, neutrophil-derived IL-1 $\beta$ may contribute to a cytokine milieu that supports the generation of Th17 cells and inflammatory IL- $17^{+}$Tregs (37). We also observed reduced survival in neutrophils coincubated with GBS, which may be relevant to our data given that apoptotic signaling in neutrophils involves IL-1 $\beta$ release and Th17-type responses (38). In contrast, neutrophil-mediated induction of Th17 cells does not appear to involve IL-6 (29), another key Th17propagating cytokine (35). Studies are currently underway to characterize the neutrophil-derived mediator(s) involved in neonatal $\mathrm{CD} 4^{+} \mathrm{T}$-cell differentiation and to more fully define the underlying mechanisms.

The key observation made in the present paper, i.e., that GBS can stimulate neonatal neutrophils to promote Th1- and Th17-type responses, adds to existing knowledge of a role for GBS in the induction of neonatal inflammatory injury, such as meningitis (39). Conversely, GBS has also been shown to suppress neutrophil responses through molecular mimicry mediated by Siglec-9 (ref. (20)), although whether this or related mechanisms are important in neonates is unclear. We reported diminished expression of Siglec-9 and SHP-1 as well as enhanced basal signaling via PI3K/Akt in neonatal neutrophils (40). These findings suggest a possible curtailment of GBS-mediated suppression in neonatal relative to adult neutrophils, which would be consistent with the greater GBS-mediated induction of inflammatory responses in neonatal immune cells observed in the present study and reported by others (22-24). These data suggest that severe GBS infection in fetuses and neonates could promote immune perturbations that simultaneously provoke inflammation while suppressing protective responses, possibilities deserving further investigation.

In summary, our present data suggest a compelling role for neonatal neutrophils in mediating Th cell responses through a combination of innate and adaptive immune mechanisms. While the resulting transformations could potentially facilitate neonatal protective immune responses against GBS (31); conversely, exaggerated inflammatory responses might amplify the pathologic mechanisms associated with neonatal infection to promote a vicious cycle of inflammation (4). Despite the inherent limitations of in vitro studies, our data provide important new information that advances understanding of inflammatory mechanisms in the vulnerable neonatal population and that sets the stage for future investigations.

\section{ACKNOWLEDGMENTS}

We gratefully acknowledge the SSM Health St Mary's Health Center Labor and Delivery physicians and staff and the SSM Health St Louis Cord Blood Bank for their assistance in obtaining samples for our studies. We thank Ryan Weisert, Linda Winter, Elizabeth Walker, and Jay McQuillan for their expert technical skills and much appreciated assistance. We are indebted to Morven Edwards for providing us with critical reagents and for helpful discussion.

\section{STATEMENT OF FINANCIAL SUPPORT}

This work was supported in part by the Saint Louis University Department of Pediatrics, the Cardinal Glennon Foundation (to J.M.K.), and the National Institutes of Health (AI094478, to J.M.K. and G.P.)

Disclosure: The authors declare no conflict of interest.

\section{REFERENCES}

1. Baker CJ. Early onset group B streptococcal disease. J Pediatr 1978;93: 124-5.

2. Edwards MS, Baker CJ. Group B streptococcal infections in elderly adults. Clin Infect Dis 2005;41:839-47.

3. Phares CR, Lynfield R, Farley MM, et al. Epidemiology of invasive group B streptococcal disease in the United States, 1999-2005. JAMA 2008;299: 2056-65.

4. Henneke P, Berner R. SIRS and group-B streptococcal sepsis in newborns: pathogenesis and perspectives in adjunctive therapy. Semin Fetal Neonatal Med 2006;11:333-42.

5. Mohammadi N, Midiri A, Mancuso G, et al. Neutrophils directly recognize Group B streptococci and contribute to interleukin-1beta production during infection. PLoS ONE 2016;11:e0160249.

6. De Paepe ME, Friedman RM, Gundogan F, Pinar H, Oyer CE. The histologic fetoplacental inflammatory response in fatal perinatal group B-streptococcus infection. J Perinatol 2004;24:441-5.

7. Kolaczkowska E, Kubes P. Neutrophil recruitment and function in health and inflammation. Nat Rev Immunol 2013;13:159-75. 


\section{Articles | Lin et al.}

8. Lawrence SM, Corriden R, Nizet V. Age-appropriate functions and dysfunctions of the neonatal neutrophil. Front Pediatr 2017;5:23.

9. Contrino J, Krause PJ, Slover N, Kreutzer D. Elevated interleukin-1 expression in human neonatal neutrophils. Pediatr Res 1993;34:249-52.

10. Thornton NL, Cody MJ, Yost CC. Toll-like receptor $1 / 2$ stimulation induces elevated interleukin-8 secretion in polymorphonuclear leukocytes isolated from preterm and term newborn infants. Neonatology 2012;101: 140-6.

11. Pelletier M, Maggi L, Micheletti A, et al. Evidence for a cross-talk between human neutrophils and Th17 cells. Blood 2010;115:335-43.

12. Black A, Bhaumik S, Kirkman RL, Weaver CT, Randolph DA. Developmental regulation of Th17-cell capacity in human neonates. Eur J Immunol 2012;42:311-9.

13. Rito DC, Viehl LT, Buchanan PM, Haridas S, Koenig JM. Augmented Th17-type immune responses in preterm neonates exposed to histologic chorioamnionitis. Pediatr Res 2016;81:639-45.

14. Gleditsch DD, Shornick LP, Van SJ, Gressens P, Weisert RP, Koenig JM. Maternal inflammation modulates infant immune response patterns to viral lung challenge in a murine model. Pediatr Res 2014;76:33-40.

15. Clarke D, Letendre C, Lecours MP, et al. Group B Streptococcus induces a robust IFN-gamma response by $\mathrm{CD} 4(+) \mathrm{T}$ cells in an in vitro and in vivo model. J Immunol Res 2016;2016:5290604.

16. Patras KA, Rosler B, Thoman ML, Doran KS. Characterization of host immunity during persistent vaginal colonization by Group B Streptococcus. Mucosal Immunol 2015;8:1339-48.

17. Zheng Y, Sun L, Jiang T, Zhang D, He D, Nie H. TNFalpha promotes Th17 cell differentiation through IL-6 and IL-1beta produced by monocytes in rheumatoid arthritis. J Immunol Res 2014;2014:385352.

18. Tecchio C, Micheletti A, Cassatella MA. Neutrophil-derived cytokines: facts beyond expression. Front Immunol 2014;5:508.

19. Albanyan EA, Vallejo JG, Smith CW, Edwards MS. Nonopsonic binding of type III Group B Streptococci to human neutrophils induces interleukin- 8 release mediated by the p38 mitogen-activated protein kinase pathway. Infect Immun 2000;68:2053-60.

20. Carlin AF, Uchiyama S, Chang YC, Lewis AL, Nizet V, Varki A. Molecular mimicry of host sialylated glycans allows a bacterial pathogen to engage neutrophil Siglec-9 and dampen the innate immune response. Blood 2009;113:3333-6.

21. Ye J, Su X, Hsueh EC, et al. Human tumor-infiltrating Th17 cells have the capacity to differentiate into IFN-gamma+ and FOXP3+ T cells with potent suppressive function. Eur J Immunol 2011;41:936-51.

22. Vallejo JG, Baker CJ, Edwards MS. Interleukin-6 production by human neonatal monocytes stimulated by type III group B streptococci. J Infect Dis $1996 ; 174: 332-7$.

23. Berner R, Welter P, Brandis M. Cytokine expression of cord and adult blood mononuclear cells in response to Streptococcus agalactiae. Pediatr Res 2002;51:304-9.

24. Sinnott BD, Park B, Boer MC, Lewinsohn DA, Lancioni CL. Direct TLR-2 costimulation unmasks the proinflammatory potential of neonatal CD4+ T cells. J Immunol 2016;197:68-77.
25. Kollmann TR, Kampmann B, Mazmanian SK, Marchant A, Levy O. Protecting the newborn and young infant from infectious diseases: lessons from immune ontogeny. Immunity 2017;46:350-63.

26. Guenova E, Skabytska Y, Hoetzenecker W, et al. IL-4 abrogates T(H)17 cell-mediated inflammation by selective silencing of IL-23 in antigenpresenting cells. Proc Natl Acad Sci USA 2015;112:2163-8.

27. Nyirenda MH, Sanvito L, Darlington PJ, et al. TLR2 stimulation drives human naive and effector regulatory $\mathrm{T}$ cells into a Th17-like phenotype with reduced suppressive function. J Immunol 2011;187: 2278-90.

28. Wennekamp J, Henneke P. Induction and termination of inflammatory signaling in group B streptococcal sepsis. Immunol Rev 2008;225: $114-27$.

29. Abi Abdallah DS, Egan CE, Butcher BA, Denkers EY. Mouse neutrophils are professional antigen-presenting cells programmed to instruct Th1 and Th17 T-cell differentiation. Int Immunol 2011;23:317-26.

30. Thewissen M, Damoiseaux J, van de Gaar J, Tervaert JW. Neutrophils and T cells: bidirectional effects and functional interferences. Mol Immunol 2011;48:2094-101.

31. McGeachy MJ, McSorley SJ. Microbial-induced Th17: superhero or supervillain? J Immunol 2012;189:3285-91.

32. Wynn JL, Wilson CS, Hawiger J, et al. Targeting IL-17A attenuates neonatal sepsis mortality induced by IL-18. Proc Natl Acad Sci USA 2016;113:E2627-35.

33. Egan CE, Sodhi CP, Good M, et al. Toll-like receptor 4-mediated lymphocyte influx induces neonatal necrotizing enterocolitis. J Clin Invest 2016;126:495-508.

34. Blatner NR, Mulcahy MF, Dennis KL, et al. Expression of RORgammat marks a pathogenic regulatory $\mathrm{T}$ cell subset in human colon cancer. Sci Transl Med 2012;4:164ra159.

35. Acosta-Rodriguez EV, Napolitani G, Lanzavecchia A, Sallusto F. Interleukins lbeta and 6 but not transforming growth factor-beta are essential for the differentiation of interleukin 17 -producing human $\mathrm{T}$ helper cells. Nat Immunol 2007;8:942-9.

36. Fong ON, Chan KY, Leung KT, et al. Expression profile of cord blood neutrophils and dysregulation of HSPA1A and OLR1 upon challenge by bacterial peptidoglycan. J Leukoc Biol 2014;95:169-78.

37. Rueda CM, Presicce P, Jackson CM, et al. Lipopolysaccharide-induced chorioamnionitis promotes IL-1-dependent inflammatory FOXP3+ CD4 + T cells in the fetal rhesus macaque. J Immunol 2016;196:3706-15.

38. Su CC, Lin HC, Lin YP, Shan YS, Yang BC. Expression of Th17-related genes in PHA/IL-2-activated human T cells by Fas signaling via caspase1- and Stat3-dependent pathway. Cell Immunol 2013;281:101-10.

39. Doran KS, Liu GY, Nizet V. Group B streptococcal beta-hemolysin/ cytolysin activates neutrophil signaling pathways in brain endothelium and contributes to development of meningitis. J Clin Invest 2003;112: 736-44.

40. Rashmi R, Bode BP, Panesar N, et al. Siglec- 9 and SHP-1 are differentially expressed in neonatal and adult neutrophils. Pediatr Res 2009;66: 266-71. 